\title{
Sémiologie des handicaps en médecine physique et de réadaptation
}

\section{Clinical aspects of disability in physical medicine and rehabilitation}

\section{J.-M. Wirotius}

(C) Springer-Verlag France 2011

À signaler à tous nos collègues médecins MPR et professionnels de santé : la parution aux éditions Lambert-Lucas de l'excellent ouvrage de notre collègue le Pr Jean-Michel Wirotius sur le thème Sémiologie des handicaps en médecine physique et de réadaptation.

Ce livre, préfacé par Jacques Fontanille de l'institut universitaire de France, vient combler un vide linguistique dans la démarche de « mise en mots » du handicap et dans la recherche des significations en réadaptation, pour permettre à tous les professionnels de santé de partager un même langage.

Jean-Michel Wirotius est le mieux placé dans cette approche du « discours des corps handicapés » du fait de sa double formation médicale (chef du service de MPR de Brive) et linguistique (centre de recherches sémiotiques EA3648 du département des sciences du langage, université de Limoges, F-87000 Limoges).

ISBN : 978-2-915806-73-1, 230 pages, $45 €$. 